\title{
All-Optical 2R Regeneration Based on Polarization Rotation in a Linear Optical Amplifier
}

\author{
Mingshan Zhao, Jan De Merlier, Geert Morthier, Senior Member, IEEE, and Roel Baets, Senior Member, IEEE
}

\begin{abstract}
All-optical 2R-regeneration based on polarization rotation in a single semiconductor linear optical amplifier is experimentally demonstrated. An extinction ratio (ER) improvement of $15 \mathrm{~dB}$ has been obtained for an input ER of $5 \mathrm{~dB}$ under static operation. With a degraded input signal, a receiver-sensitivity improvement of $>3 \mathrm{~dB}$ at a bit error rate of $10^{-9}$ was found for $2.5 \mathrm{~Gb} / \mathrm{s}$. Regenerative capability for $10 \mathrm{~Gb} / \mathrm{s}$ has also been verified.
\end{abstract}

Index Terms-All-optical regeneration, optical communication, optical pulse shaping, polarization, semiconductor optical amplifiers (SOAs).

\section{INTRODUCTION}

A LL-OPTICAL signal processing is considered to be of key importance in future all-optical networks. Semiconductor optical amplifiers (SOAs) are very attractive in switching, gating, wavelength conversion, and regeneration due to their optical gain, strong nonlinear behavior, and integratability in photonic circuits. Cross-gain and cross-phase modulation and four-wave mixing have been widely used in all-optical signal processing [1]-[3]. Recently, it has been proposed to make use of polarization rotation induced by nonlinear birefringence in an SOA [4] in all-optical switching [5] and wavelength conversion [6]. In this letter, we experimentally demonstrate all-optical 2R-regeneration based on such kind of induced polarization rotation in a single semiconductor linear optical amplifier (LOA) [7]. An extinction-ratio (ER) improvement of $15 \mathrm{~dB}$ has been obtained with an input ER of $5 \mathrm{~dB}$ under static operation. With a degraded input signal, a receiver-sensitivity improvement of more than $3 \mathrm{~dB}$ for $2.5 \mathrm{~Gb} / \mathrm{s}$ and zero power penalty for $10 \mathrm{~Gb} / \mathrm{s}$ at a bit error rate (BER) of $10^{-9}$ has been found.

\section{PRINCIPLE OF OPERATION}

The regenerator basically consists of an LOA followed by a polarization controller and a polarizer, as shown in Fig. 1. The operation of this regenerator is based on the polarization rotation induced by nonlinear effective birefringence in the LOA. While in the linear regime the birefringence is independent of the input-power level, it changes rapidly when the LOA becomes saturated. The transverse electric (TE) and transverse

Manuscript received July 18, 2002; revised October 2, 2002. This work was supported by the Belgian Federal Office for Scientific, Technical and Cultural Affairs under the Research Network IAP V-18 and the PHOTON Network.

The authors are with the Department of Information Technology, Ghent University-IMEC, B-9000 Gent, Belgium (e-mail: mszhao@ intec.rug.ac.be).

Digital Object Identifier 10.1109/LPT.2002.806091

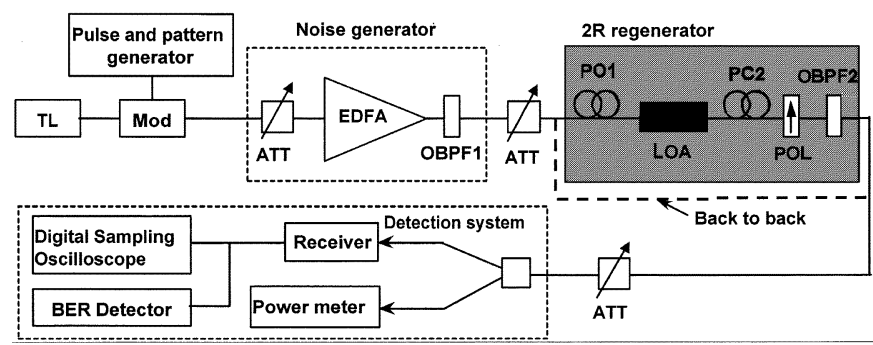

Fig. 1. Experimental setup and structure of the 2R regenerator. TL: tunable laser, ATT: variable attanuator, Mod: modulator, PC: polarization controller, Pol: polarizer, OBPF: optical bandpass filter.

magnetic (TM) effective indices at position $z$ in the active region can be given as

$$
n_{\mathrm{TE} / \mathrm{TM}}(z)=n_{0 \mathrm{TE} / \mathrm{TM}}+\Gamma_{\mathrm{TE} / \mathrm{TM}} N(z)\left(\frac{\partial n}{\partial N}\right)
$$

where $n_{0}$ is the effective refractive index of the waveguide for zero-free carrier density, $\Gamma$ is the confinement factor, and $(\partial n / \partial N)$ is the rate of change of active layer refractive index with the carrier density $N(z)$. The local effective index is different for the TM and TE components of the optical signal owing to the TE/TM asymmetry in both the confinement factors and the unperturbed effective refractive indices of the LOA. Thus, the LOA exhibits an effective birefringence that changes with the carrier density. Although a reasonably polarization-independent gain is technologically achievable, a total suppression of this birefringence is virtually impossible to obtain. In the linear regime, the gain of the LOA is clamped and both the free carrier density and the total photon density (photon density of both laser and optical signal) are constant and thus the birefringence remains unchanged when the input-power level varies. Once the linear power range is exceeded, the gain, and thus the carrier density, will drop rapidly and a significant change in the effective birefringence will thus result.

For a linearly polarized injected light beam, the state of polarization of the output from the LOA will be different from that at the input but it will not change with varying input-power level in the linear regime. Hence, by linearizing the polarization at the output of the LOA with a polarization controller and by setting the polarizer so as to block the output beam, a very low output power (or a logical "0") is obtained below the input saturation power of the LOA. Beyond the saturation power of the LOA, the state of polarization at the output of the LOA is changed due to a change in the birefringence. Both the orientation (i.e., azimuth) and the ellipticity of the polarization vary rapidly with the input power. The polarizer can no longer block the output from the LOA. Hence, a high power level (or a logical "1") is obtained 
at the output of the polarizer. At the same time, the LOA is saturated and its gain quickly drops with increasing input power, and thus the high power level of the output becomes saturated. As a result, an optical regeneration is realized. The polarization controller before the LOA is added to adjust the initial polarization of the input signal beam, and thus to get an optimum polarization rotation effect [4].

In addition to the induced birefringence effects mentioned above, TE/TM conversion effect has been shown to be another contributor to the polarization rotation in SOAs [4]. The association of induced birefringence and induced TE/TM conversion accentuates the polarization nonlinearity of the device. The former effect induces a modification of the state of polarization when light, with an input polarization different from TE or $\mathrm{TM}$, propagates through the device. The latter effect results in some polarization rotation even for a TE and TM input mode. Both modifications vary with the intensity of the light beyond the linear regime.

The advantages of this new scheme of all-optical 2R regeneration are its immunity against small signal distortion in the " 0 " level and a potentially large ER improvement. This results from the flat gain response for small signal powers and the sudden drop in gain as soon as the linear power range is exceeded.

\section{EXPERIMENTAL RESULTS AND DisCUSSION}

The experimental setup used for demonstrating optical regeneration is shown in Fig. 1. A tunable laser source (Model Tunics-plus, Photonetics), an external modulator, and a pulse pattern generator (Advantest D3186) are combined and used as a transmitter. A variable attenuator and an erbium-doped fiber amplifier are used to control the signal-to-noise ratio at the input so as to simulate the degradation of the signal. The ER of the input data signal is controlled by changing the driving pulse amplitude (i.e., electrical pulse-signal amplitude) of the external modulator. The LOA (Genoa) is biased with $250 \mathrm{~mA}$. The optical bandpass filter (OBPF2) after the 2R regenerator has a 3-dB bandwidth of $0.3 \mathrm{~nm}$ and is used to reduce the amplified spontaneous emission from the LOA. The polarizer has an ER of $30 \mathrm{~dB}$. A Digital Sampling Oscilloscope (Tektronix 11 801B) and an Error Detector (Advantest D3286) are used in the detection system for measuring the eye diagrams and BER, respectively. The regeneration characteristics of the $2 \mathrm{R}$ regenerator are investigated for static operation and dynamic operation at both $2.5 \mathrm{~Gb} / \mathrm{s}$ [nonreturn-to-zero (NRZ), $2^{9}-1$ pseudorandom binary sequence (PRBS)] and $10 \mathrm{~Gb} / \mathrm{s}\left(\mathrm{NRZ}, 2^{31}-1\right.$ PRBS).

Fig. 2 gives the measured static transfer characteristic of the $2 \mathrm{R}$ regenerator. It can be seen that for a logical " 0 " (low power level) quasi-perfect regeneration is achieved. An ER improvement of $19 \mathrm{~dB}$ can be obtained for the input ER of $10 \mathrm{~dB}$. Even for a very low input ER (e.g., $5 \mathrm{~dB}$ ) an ER improvement of $15 \mathrm{~dB}$ can be achieved.

The regenerative capabilities of the regenerator under dynamic operation at $2.5 \mathrm{~Gb} / \mathrm{s}$ are demonstrated in Fig. 3 and 4. Fig. 3 shows the eye diagrams with and without the $2 \mathrm{R}$ regenerator for different input ERs, while Fig. 4 shows BER measurement results. Clearly, the input signal is regenerated. The noise background at the logical " 0 " in the input signal is reduced. The

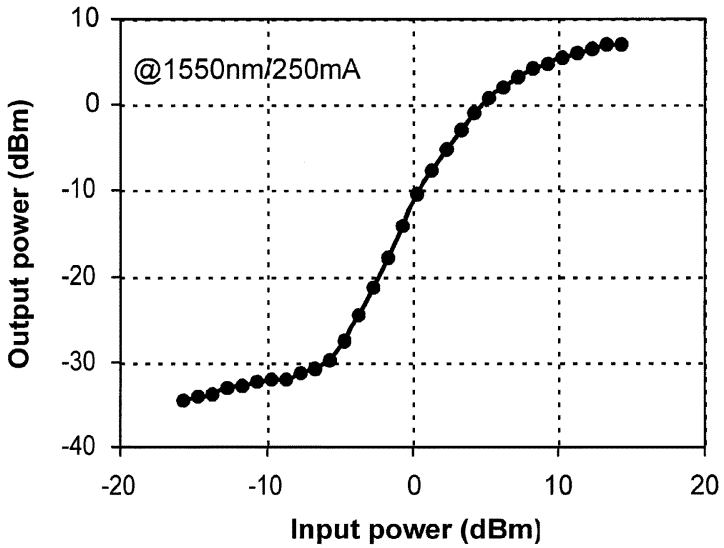

Fig. 2. Measured static regeneration characteristic.
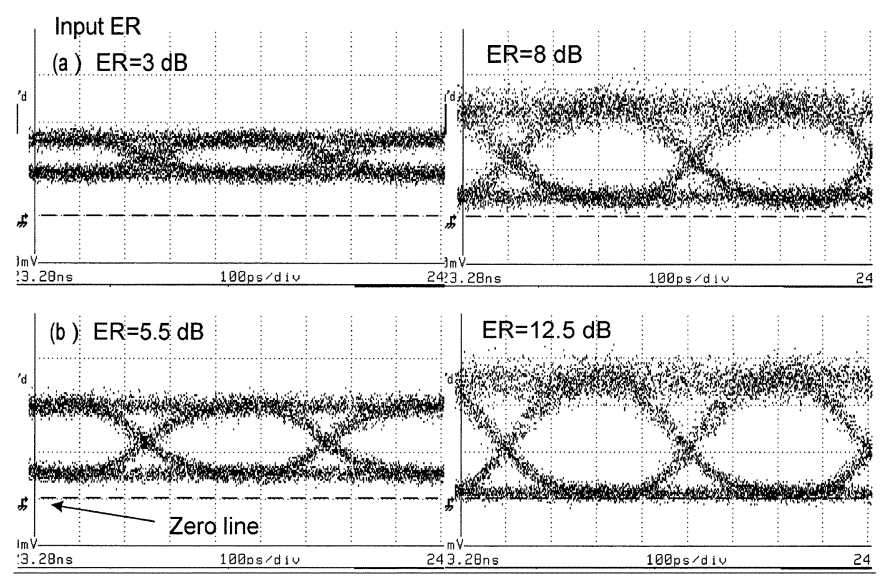

Fig. 3. Eye diagrams with and without $2 \mathrm{R}$ regenerator for different input signal ER.

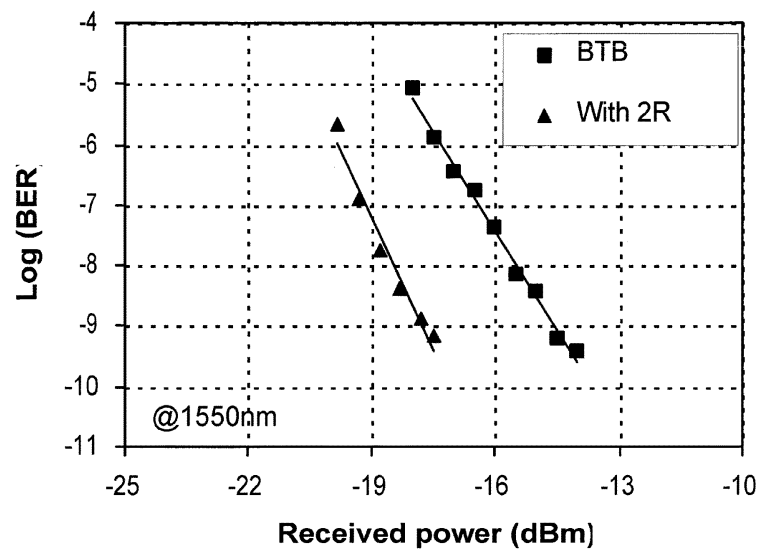

Fig. 4. BER measurement results with and without $2 \mathrm{R}$ at $2.5 \mathrm{~Gb} / \mathrm{s}$. Input ER: $3 \mathrm{~dB}$. Input power: $3 \mathrm{dBm}$.

eyes becomes much more open after the $2 \mathrm{R}$ regenerator. A significant improvement of the ER is demonstrated. For an input signal with a 5.5-dB ER, a 7-dB improvement in ER is obtained. Even for a very deteriorated input signal with an extinction of $3 \mathrm{~dB}$, an ER improvement of $5 \mathrm{~dB}$ is still achieved. Fig. 4 contains the results from the BER measurement, and shows that a receiver-sensitivity improvement of $3.3 \mathrm{~dB}$ at a BER of $10^{-9}$ can be achieved with the $2 \mathrm{R}$ regenerator proposed above. 


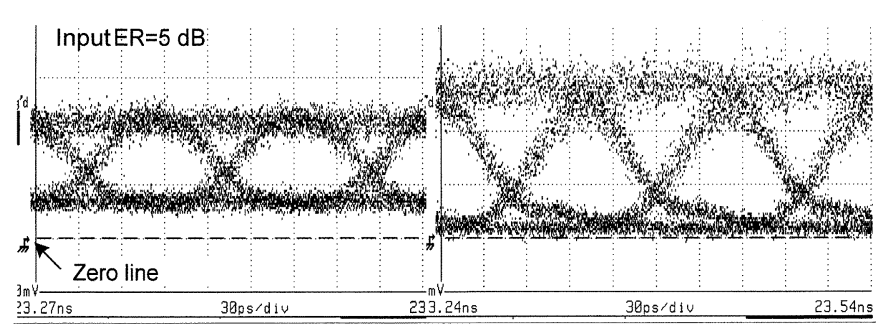

Fig. 5. Eye diagrams with and without $2 \mathrm{R}$ regenerator for $10 \mathrm{~Gb} / \mathrm{s}$.

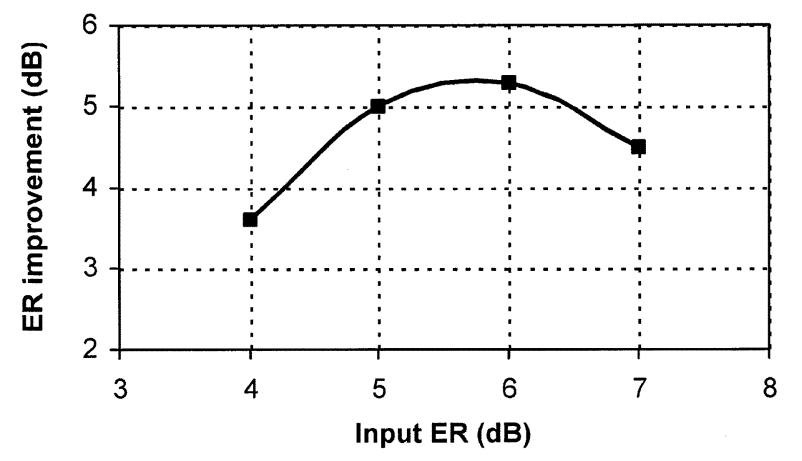

Fig. 6. ER improvement as a function of input ER for $10 \mathrm{~Gb} / \mathrm{s}$, input power: $5 \mathrm{dBm}$.

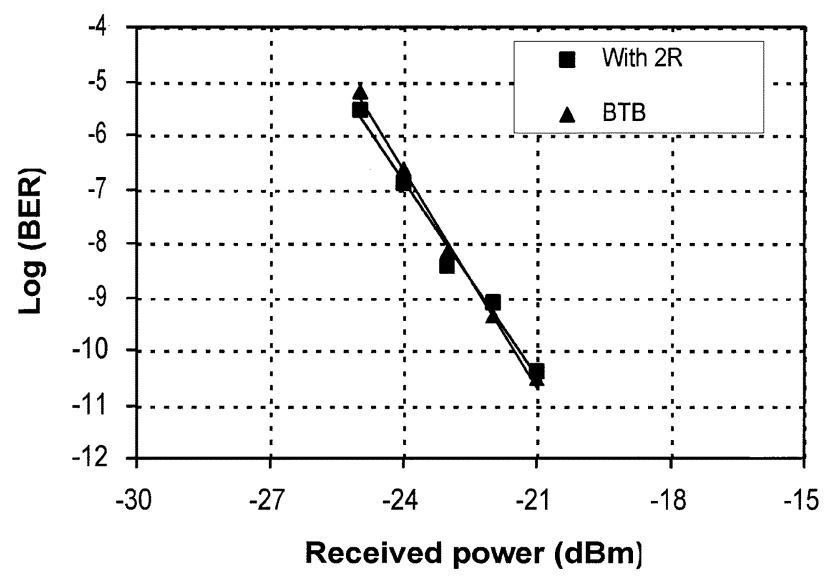

Fig. 7. BER measurement results with and without $2 \mathrm{R}$ at $10 \mathrm{~Gb} / \mathrm{s}$. Input power: $5 \mathrm{dBm}$. Input ER: $5 \mathrm{~dB}$.

Experiments for $10 \mathrm{~Gb} / \mathrm{s}$ (NRZ, $2^{31}-1$ PRBS) have also been carried out. Due to the low sensitivity of the receiver (HP11982A), an optical preamplifier was used after the 2R. Improvements of both eye diagram and ER are observed, as seen in Fig. 5 and 6, showing a clear regenerative capability of the $2 \mathrm{R}$ regenerator at $10 \mathrm{~Gb} / \mathrm{s}$. For an input signal with $6-\mathrm{dB}$ $\mathrm{ER}$, an ER improvement of $>5 \mathrm{~dB}$ has been obtained. The BER measurement results are given in Fig. 7. A power penalty of $0 \mathrm{~dB}$ is found and no receiver-sensitivity improvement has been obtained. This is due to a pattern effect induced by polarization relaxation at the trailing edge of the signal pulse, as seen in Fig. 5. Actually, the state of polarization at the output of the LOA when it is just out of saturation at the trailing edge of the signal pulse is different from that before it gets into saturation at the leading edge. It takes a time (about $60 \mathrm{ps}$ for the LOA used in our experiments) to recover the state of polarization at " 0 " levels. This effect could be reduced with a specifically designed LOA with high speed and weak polarization relaxation.

The decrease in required received power in order to obtain a BER of $10^{-9}$ presented in the above sections implies that the noise is redistributed and decreased. Furthermore, simultaneous ER improvement has been obtained. The induced chirp of the device has not been measured but due to the fact that a passthrough scheme works inherently in the noninverted scheme and the component is based on self-gain modulation, a red shift will occur at the leading edge and a blue shift at the trailing edge. This is the desired behavior for a propagating pulse. Based on these arguments, we can state that this device should be able to work in a cascade in order to have signal propagated over a large distance.

It is clear, however, that control of input polarization is required in this new scheme, which may be disadvantageous in some circumstances. But it could be extended to an input polarization insensitive $2 \mathrm{R}$ regenerator with some polarization-diversity scheme.

\section{CONCLUSION}

A novel all-optical 2R regenerator has been demonstrated utilizing polarization rotation induced by nonlinear birefringence in a single LOA. Significant improvement in ER has been found. For a degraded input signal, a receiver-sensitivity improvement of over $3 \mathrm{~dB}$ at a BER of $10^{-9}$ is achieved for $2.5 \mathrm{~Gb} / \mathrm{s}$. The regenerative capability at $10 \mathrm{~Gb} / \mathrm{s}$ has also been verified. The features of simple configuration, stable operation, and high regenerative capabilities make this new scheme a promising technique for all-optical regeneration in future optical networks.

\section{ACKNOWLEDGMENT}

The authors would like to thank their colleagues at Genoa for useful discussions and for providing the LOAs.

\section{REFERENCES}

[1] K. E. Stubkjaer, "Semiconductor optical amplifier-based all-optical gates for high-speed optical processing," IEEE J. Select. Topics Quantum Electron., vol. 6, pp. 1428-1435, Nov./Dec. 2000.

[2] O. Leclerc, B. Lavigne, and D. Chiaroni, "All-optical regeneration: Principles and WDM implementation," in Optical Fiber Telecommunications IVA (Components), I. P. Kaminow and T. Li, Eds. San Diego, CA: Academic, 2002, pp. 732-783.

[3] M. Zhao, G. Morthier, and R. Baets, "Demonstration of extinction ratio improvement from 2 to $9 \mathrm{~dB}$ and intensity noise reduction with the MZIGCSOA all-optical 2R regenerrator," IEEE Photon. Technol. Lett., vol. 14, pp. 992-994, July 2002.

[4] H. Soto, D. Erasme, and G. Guekos, "Cross-polarization modulation in semiconductor optical amplifiers," IEEE Photon. Technol. Lett., vol. 11, pp. 970-972, Aug. 1999.

[5] D. Erasme, H. Soto, and G. Guekos, "All-optical switching and XORgating using cross-polarization modulation in a semiconductor optical amplifier," presented at the CLEO/Europe, Nice, France, Sept. 2000, Paper CtuD3.

[6] M. F. C. Stephens, M. Asghari, R. V. Penty, and I. H. White, "Demonstration of ultrafast all-optical wavelength conversion utilizing birefringence in semiconductor optical amplifiers," IEEE Photon. Technol. Lett., vol. 9, pp. 449-451, Apr. 1997.

[7] D. A. Francis, S. P. DiJaili, and J. D. Walker, "A sinlge-chip linear optical amplifier," presented at the OFC'2001, Anaheim, CA, Mar. 2001, Paper PD-13. 